\title{
A Singular web service for geometric computations
}

\author{
Francisco Botana • Zoltán Kovács
}

Received: date / Accepted: date

The final publication is available at Springer via http://dx.doi.org/10.1007/s10472014-9438-2

Abstract Outsourcing algebraic computations in dynamic geometry is a possible strategy used when software distribution constraints apply. Either if the target user machine has hardware limitations, or if the computer algebra system cannot be easily (or legally) packaged inside the geometric software, this approach can solve current shortcomings in dynamic environments.

We report the design and implementation of a web service using Singular, a program specialized in ideal theory and commutative algebra. Besides its canonical address, a virtual appliance and a port to a low-cost ARM based computer are also provided. Any interactive geometric environment can then outsource computations where Singular is used, and incorporate their results into the system. In particular, we illustrate the capabilities of the web service by extending current abilities of GeoGebra to deal with algebraic loci and envelopes by means of a recent algorithm for studying parametric polynomial systems.

First author partially supported by the Spanish "Ministerio de Economía y Competitividad" and by the European Regional Development Fund (ERDF), under the Project MTM201125816-C02-02.

\section{F. Botana}

Dept. Applied Mathematics I, University of Vigo, Campus A Xunqueira, 36005 Pontevedra, Spain

Tel.: +34-986-801900

Fax: +34-986-801901

E-mail: fbotana@uvigo.es

Z. Kovács

Dept. Mathematics Education, Johannes Kepler University, Altenberger Strasse 54, 4040 Linz, Austria

Tel.: +43-732-2468-6881

E-mail: zoltan@geogebra.org 
Keywords Web services - Dynamic geometry - Parametric polynomial systems · Geometric loci $\cdot$ Envelopes

Mathematics Subject Classification (2000) MSC2010 68W30 • MSC2010 $68 \mathrm{~T} 35$

\section{Introduction}

Dynamic geometry (DG) software refers to computer programs where accurate geometric constructions can be done. Some elements of a construction can be dragged and, as they do, the whole diagram moves accordingly, preserving essential relations and constraints. Consider, for instance, the ruler and compass construction of the midpoint of a pair of points. Its DG construction involves two free points, $A$ and $B$, and their midpoint. Dragging $A$ (or $B$ ) the midpoint also moves but maintaining its defining characteristic, while if the user tries to drag the midpoint nothing happens or the entire contruction is moved.

Although a remote antecedent of this paradigm can be traced back to Sutherland's Sketchpad [1], there is consensus about considering Cabri Geometry ${ }^{1}$ and The Geometer's Sketchpad ${ }^{2}$ as the environments marking the birth of massive use of DG for mathematics education. DG environments, apart from their educational uses, fastly attracted the attention of the community of automated deduction in geometry. Coordinate-based approaches for automatic proving, such as the method of $\mathrm{Wu}$ and the Groebner bases method, have been successfully used during the last thirty years. DG software incorporating Wu's method are MMP/Geometer [2] and Geometry Expert [3], to mention a few. Concerning the Groebner basis method a proposal for linking DG environments with this technique was given in [4] and realized in academic prototypes (see for example $[5,6]$ ).

Meanwhile, a new DG system was proposed in 2001. GeoGebra ${ }^{3}$ is an open source environment written in Java aiming to support under a common tool the elementary study of algebra and geometry. The software rapidly spread throughout the math educational world, and has become a collaborative project with impressive figures regarding users, languages versions and web visitors. Although initial plans of GeoGebra developers were distributing the software as a whole, the authors and other colleagues have considered enriching GeoGebra through the remote access to specialized software, mainly for tasks related to automating proof and discovery of geometric properties. One tool is Singular [7], specially well suited for dealing with ideal theory and commutative algebra. Section 2 describes a web service using Singular. Although designed with GeoGebra in mind, it can be used with any DG system. As an illustration of its possibilities, Section 3 gives an account of a pair of GeoGebra commands where the web service plays an outstanding role. By using

\footnotetext{
1 http://cabri.com

2 http://www.keycurriculum.com/products/sketchpad

3 http://www.geogebra.org
} 
a recent algorithm for solving parametric polynomial systems, GroebnerCover [8], an existing GeoGebra command for computing the algebraic description of geometric loci is improved and a new one dealing with envelopes of curves is implemented. Both commands will be part of the forthcoming version 5.0 of GeoGebra.

\section{The SingularWS web service}

Singular is mostly written in $\mathrm{C}++$. Embedding it into GeoGebra would be theoretically possible. GeoGebra is shipped as two kinds of main but completely different platforms: the classical, desktop based version, running in a Java Virtual Machine (JVM) can include embedded systems written in different languages than Java. The standard technology here is Java Native Interface (JNI, [9]). Unfortunately, the Java standard is no longer well supported in the recent mobile devices including tablets and smartphones.

This is why GeoGebra has been moving towards a new platform, HTML5, since it is the de facto standard of many of the present developments worldwide. The HTML5 based web version of GeoGebra [10] may also include a compiled version of Singular via a $\mathrm{C}++$ to JavaScript or Native Client compiler. This kind of embedding has already been succeeded with the Giac CAS, also being written in $\mathrm{C}++$. See [12] for more details. Despite the theoretical possibility, for size and simplicity reasons using Singular from GeoGebra was definitely planned and implemented as an external service. According to site documentation $^{4}$, the minimal Windows installer of Singular 3.1.6 is 36 MB of length which is about four times bigger than GeoGebra itself. For comparison, Giac can be compressed to fit in $4 \mathrm{MB}$ as a native library and in $7 \mathrm{MB}$ as JavaScript code. On the other hand, Giac is rather a general purpose CAS and lacks of the algorithm used throughout this note.

As a conclusion, we decided to use an outsourced solution by installing a Singular instance on a machine accessible via Internet [11]. Our demonstrational public server (also used in GeoGebra 5.0 by default) is currently at singularws.idm.jku.at, what is more, its full software content is also available for downloading at [13] (375 MB .zip file). Thus a teacher has two options: he either uses our default public server, or has the opportunity to download the ISO image of SingularWS, install it into VirtualBox and set the remote machine to the locally installed Singular web service (SingularWS) instance. This latter option gives more flexibility for heavy computations. A detailed documentation of this technical process can be read in [14] and [15]. The end user (the student or the system administrator of the classroom) needs to add the command line argument --SingularWS=remoteURL: http://... when using GeoGebra as in the second option; here . . . is the IP address (or fully qualified domain name) of the SingularWS instance.

4 http://www.singular.uni-kl.de/index.php/singular-download/install-windows-single-file.html 


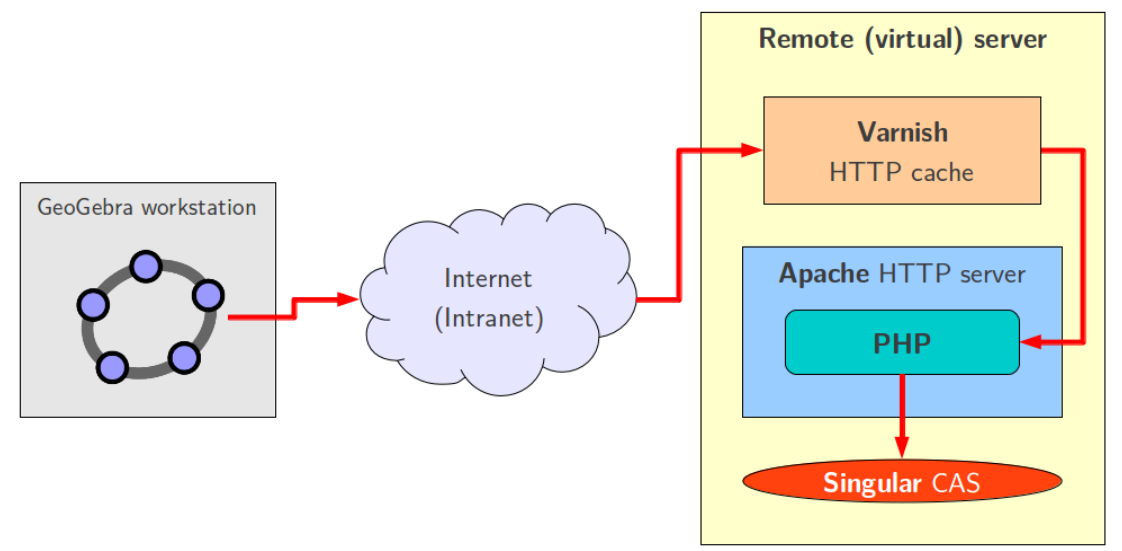

Fig. 1 GeoGebra communicates with SingularWS via the HTTP protocol. Caching layer software Varnish can also be inserted to speed up classroom usage when the same queries may be sent by more students, but only one computation is preferred for all of them. Newest version of SingularWS itself can maintain caching without any extra software required.

Besides the ISO image, a port of SingularWS for ARM architecture can also be downloaded ${ }^{5}$. This image (3 GB .zip file), specifically taylored for the low-cost Raspberry Pi computer, uses Singular through its embedding in Sage. Although computation times will rise by a factor of ten, this solution could be well suited for wireless use at schools with no Internet access and heavy budget constraints. By switching SingularWS caching on with command line argument --SingularWS=caching:true in GeoGebra, the teacher can pregenerate the heavy computations for the local SingularWS instance before the classroom showtime and the students will use the pre-computed results on their workstations in an immediate time. In this case it is suggested to use such teaching materials where the free points are constrainted to a reduced set than the whole Euclidean plane, for example to grid points (in GeoGebra Options $\triangleright$ Point Capturing $\triangleright$ Fixed to Grid).

GeoGebra communicates with Singular via a simple HTTP connection string (Fig. 1) via some simple PHP scripts. In many cases the computation time is below 10 milliseconds, and the communication (assuming an acceptable Internet bandwidth between the student machine and the Austrian university network) is below 40 milliseconds. As a result, GeoGebra with SingularWS computes a wide range of geometry problems below 50 milliseconds. On the other hand, this technology offers new perspectives in real time locus or envelope equation computations, especially when lots of such consecutive computations are needed. Online Resource 1 shows a short introductory video on

${ }^{5}$ http://193.146.36.205/pi_SingularWS_Sage5.8.img.zip 
using the LocusEquation command ${ }^{6}$ in GeoGebra 5.0 communicating with a locally installed SingularWS instance via the HTTP protocol.

We needed to restrict the Singular command set to prohibit harmful access to the SingularWS instance. Thus we made some internal modifications ${ }^{7}$ on Singular to run it in sandboxed mode by using the command line option --no-shell. This mode will be available in Singular 3.1.7. (SingularWS currently uses version 3.1.6.)

\section{GeoGebra computations via SingularWS}

Current DG environments exhibit limited abilities when manipulating symbolic geometric objects while CAS are particularly strong on such issue. Furthermore, usual development of DG software pays special attention to graphical aspects, neglecting the data structure needed for dealing with non-trivial constructions. As a consequence, academic proposals for using CAS (Mathematica, Maple, CoCoA,...) as symbolic solvers for DG systems were made repeatedly. See, for instance, $[5,6,16]$. As GeoGebra attracted a lot of attention from teachers, so were the proposals using it as a frontend for applying results coming from the automated deduction in geometry community. Nevertheless, being GeoGebra a free open source program, the use of a vast repository of knowledge and algorithms using proprietary environments poses intrinsic problems regarding its massive spread. Despite some moves of CAS corporations allowing a kind of free access to their tools (cf. Wolfram|Alpha), we think that it is not a safe approach relying on centralized closed software. Thus, SingularWS offers free open access to any existing Singular algorithm on a decentralized basis.

\subsection{The GroebnerCover algorithm}

We will use this algorithm to solve parametric polynomial systems describing GeoGebra constructions that involve loci or envelopes. Thus, we recall the main properties of the algorithm for the case of two parameters.

A locus or envelope construction is described by a polynomial system $\left\{f_{i}\left(x, y, x_{1}, \ldots, x_{n}\right): i=1, \ldots, m\right\}$, where $x, y$ are the parameters (the coordinates of a generic locus point or the variables in the family of enveloped curves), and $x_{1}, \ldots, x_{n}$ the rest of the construction variables. The solution $\mathbb{V}(\mathcal{I})=\left\{\left(x, y, x_{1}, \ldots, x_{n}\right) \in \mathbb{C}^{n+2}: \forall i, f_{i}\left(x, y, x_{1}, \ldots, x_{n}\right)=0\right\}$, where ideal $\mathcal{I}=\left\langle f_{i}\right\rangle$, can be computed by the GroebnerCover algorithm. Given the ideal $\mathcal{I} \subset \mathbb{Q}[x, y]\left[x_{1}, \ldots, x_{n}\right]$ and a monomial order, there exists a unique set of pairs $\left\{\left(S_{j}, B_{j}\right): 1 \leq j \leq s\right\}$, called Groebner cover, such that

- The segments $S_{j} \subset \mathbb{C}^{2}$ are disjoint.

\footnotetext{
6 http://wiki.geogebra.org/en/LocusEquation_Command

7 https://github.com/kovzol/Sources/commits?author=kovzol
} 
- The segments $S_{j}$ are locally closed subsets of the parameter space $\mathbb{C}^{2}$.

- Associated to each segment $S_{j}$ there is a basis $B_{j} \subset \mathbb{Q}[x, y]\left[x_{1}, \ldots, x_{n}\right]$ that specializes to the reduced Groebner basis of $\mathcal{I}$ for every pair $(x, y) \in S_{j}$.

The fact that for all elements in a segment the types of system solutions are the same (finite, infinite or no solution) helps to decide about the membership of each segment to the sought locus or envelope. Since a detailed description of the procedure is in [17] and the goal of this note is the illustration of new GeoGebra abilities by means of SingularWS, we will use it here without further explanation.

\subsection{Loci computation}

The consuetudinary DG approach for loci is also implemented in GeoGebra since its earlier version. A mover point bounded to a linear path determines another point, the tracer, whose trajectory is the sought locus. This locus is found as a linear object after sampling the mover's path, and getting a list of tracers. Heuristic rules are used to join contiguous points. See [18] for a discussion of intrinsic problems within this approach.

Reacting to the mere graphical knowledge of loci, GeoGebra, starting from version 4.2, included the new command LocusEquation. There, elimination is performed on the algebraic description of the locus construction in order to return such locus as an implicit curve. As it is well-known, such process finds the Zariski closure of the projection on the space of locus coordinates, thus possibly incorporating spurious points. As an illustration, consider the unit circle, the hyperbola $x y=1$ and a point $A$ moving along the hyperbola. The vertical projection of $A$ sweeps the circle except both poles, while elimination will return the whole unit circle, since it is the smallest variety containing the locus. The GroebnerCover algorithm proceeds a step further in the description of sets defined by first-order formulae. Instead of returning varieties, as it is the case with algebraic elimination, results are expressed by constructible sets since the base field is the complex one. In the case we are illustrating, the projection of $A$ would be declared as a locally closed set, that is, a difference of varieties: $\mathbb{V}\left(x^{2}+y^{2}-1\right) \backslash \mathbb{V}\left(y^{2}-1, x\right)$.

The current GeoGebra data structure does not allow representing constructible sets. Therefore, whenever a locus or envelope include a constructible set not expressable by means of a single variety, for instance $V_{1} \backslash V_{2}$, the system drops $V_{2}$ out and plots $V_{1}$ as a GeoGebra implicit curve. This would be the case when computing the pedal of an ellipse with respect to its center. The center is included in the variety obtained after elimination, and, although GroebnerCover takes it out, the GeoGebra implicit curve introduced in the construction includes this extra point. So, any further computation with the curve will be unsound, as can be tested trying to get again its pedal with respect to the original center. A second ad-hoc strategy deals with points. If a constructible set is a point $(a, b)$ defined by $\mathbb{V}(x-a, y-b)$ GeoGebra includes 


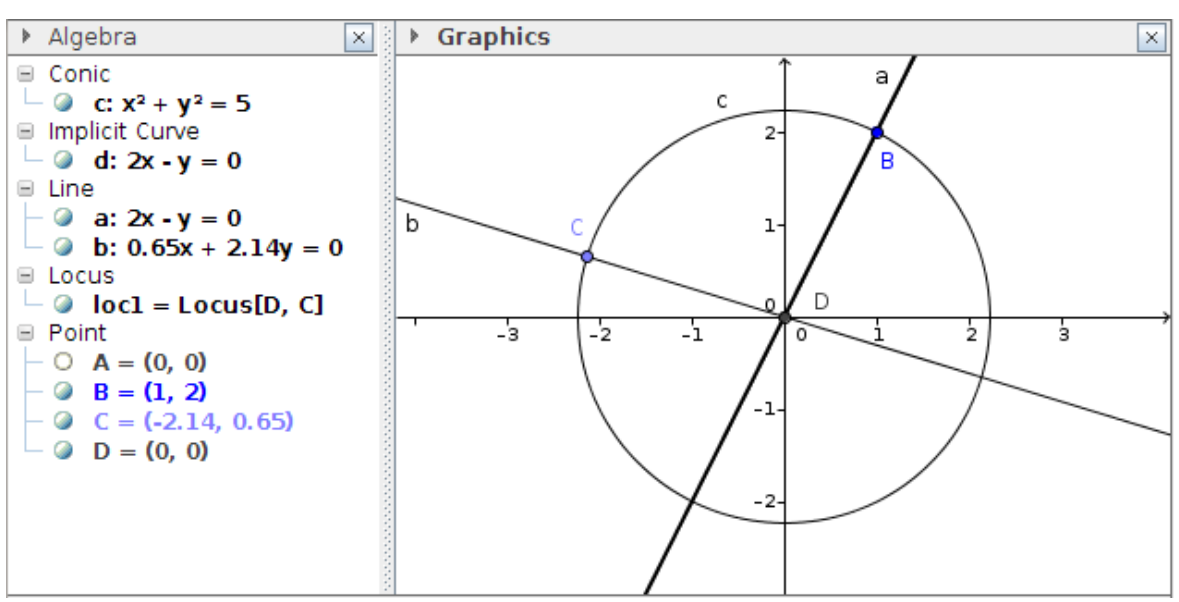

Fig. 2 The wrong locus due to degeneration (if SingularWS is not used).

it as the implicit curve $(x-a)^{2}+(y-b)^{2}=0$, despite their different algebraic meaning.

Another source of inaccuracy is due to construction degeneracies. As a simple example (Figure 2), consider a circle centered at $A$ and passing through $B$. Let $C$ be a point on the circle and define $D$ as the intersection of lines $A B$ and $A C$. It is obvious that the locus of $D$ when $C$ moves along the circle is its center, as GeoGebra finds (although the locus is not drawn, one can try to construct a point on it and check its position). Nevertheless, LocusEquation, if SingularWS is not used, will return a line as locus. This line comes from a degeneration: when $C$ coincides with $B$, also do lines $A B$ and $A C$, their intersection is not defined and the whole line is considered as a faithful part of the locus.

The locus can be derived from a parametric polynomial system, where $A(0,0), B(1,2)$. Thus, the circle is $x^{2}+y^{2}-5, C\left(x_{1}, x_{2}\right)$ satisfies $x_{1}^{2}+x_{2}^{2}-5$, and $D(x, y)$ is defined by $y-2 x$ and $y x_{1}-x x_{2}$. The Groebner cover of the ideal $\mathcal{I}=$ $\left\langle x_{1}^{2}+x_{2}^{2}-5, y-2 x, y x_{1}-x x_{2}\right\rangle$ for the degree reverse lexicographical ordering (chosen by default) consists of three segments with bases as shown in Table 1. After dropping out the segment with basis $\{1\}$ (meaning that no solution of the system exists), there are two segments where the system is solvable. The second one states that there are exactly two construction instances such that for parameters values satisfying $y=2 x$ the system has solution, namely $\left(x_{1}, x_{2}\right)=(1,2)$ or $(-1,-2)$. Finally, there are infinite solutions, the circle $x_{1}^{2}+x_{2}^{2}=5$, for parameters $x=0$ and $y=0$. We reject the line $y=2 x$ since its dimension is greater than the one spanned by the corresponding variables, and the point in third segment is declared as the locus.

The above locus construction and other illustrative examples are included in Online Resource 2. The constructions available as supplementary material 
Table 1 The Groebner cover for the circle center as a locus.

\begin{tabular}{lll}
\hline Nr. & Segment & Basis \\
\hline 1 & $\mathbb{C}^{2} \backslash \mathbb{V}(2 x-y)$ & $\{1\}$ \\
2 & $\mathbb{V}(2 x-y) \backslash \mathbb{V}(y, x)$ & $\left\{2 x_{1}-x_{2}, x_{2}^{2}-4\right\}$ \\
3 & $\mathbb{V}(y, x)$ & $\left\{x_{1}^{2}+x_{2}^{2}-5\right\}$ \\
\hline
\end{tabular}

should be opened with GeoGebra version 4.9.241.0 or greater ${ }^{8}$. Options related to SingularWS are fully documented in the release notes of the new beta version $^{9}$.

\subsection{Envelopes computation}

Whereas computing envelopes is an option present in most DG environments, GeoGebra lacks such ability. Only a simple procedure is suggested to get a rough visualization of envelopes: trace an element of the family. This approach relies on an incomplete definition of envelope and can be extremely difficult of visualizing, even for expert users. Here we use the standard envelope definition [19]: Given a family of curves $F(t, x, y)$ where $(x, y) \in \mathbb{R}^{2}$, parameter $t \in \mathbb{R}$, the envelope of $F$ is the set

$$
\left\{(x, y) \in \mathbb{R}^{2}: \exists t \in \mathbb{R}, F(t, x, y)=\partial F / \partial t(t, x, y)=0\right\},
$$

provided that, for each $t, 0$ is a regular value of $F_{t}(x, y)=F(t, x, y)$. If the family is multi-parametric, $F\left(t_{1}, \ldots, t_{n}, x, y\right)$, where the parameters are bounded by $n-1$ constraints, $\left\{g_{1}\left(t_{1}, \ldots, t_{n}\right)=0, \ldots, g_{n-1}\left(t_{1}, \ldots, t_{n}\right)=0\right\}$, the above conditions are replaced by $F=g_{1}=\ldots=g_{n-1}=0$ and

$$
\left|\begin{array}{ccc}
\partial F / \partial t_{1} & \ldots & \partial F / \partial t_{n} \\
\partial g_{1} / \partial t_{1} & \ldots & \partial g_{1} / \partial t_{n} \\
\partial g_{n-1} / \partial t_{1} & \ldots & \partial g_{n-1} / \partial t_{n}
\end{array}\right|=0
$$

Therefore, the problem, as in loci, can be posed as solving a parametric polynomial system, restricting the base field to $\mathbb{Q}$ and being aware that solutions will be found in $\mathbb{C}$. Again, generic elimination for computing envelopes could include extra branches due to degeneration and extra parts due to Zariski closures.

As an illustration (Figure 3 ) consider a circle centered at $A(4,2)$ and passing through $B(4,4)$, a point $C$ moving on it, and the line $B C$. We look for the envelope of the family of perpendicular lines to lines $B C$ passing through $C$. Tracing the lines of the family the whole plane is swept, so no clear envelope can be decided (technically, the envelope, as boundary of the region filled by the lines, is empty). Eliminating the coordinates of $C, x_{1}, x_{2}$, in the system

$$
\left(x_{1}-4\right)^{2}+\left(x_{2}-2\right)^{2}-4,\left(y-x_{2}\right)\left(x_{2}-4\right)+\left(x-x_{1}\right)\left(x_{1}-4\right),
$$

\footnotetext{
8 http://download.geogebra.org/installers/5.0

9 http://wiki.geogebra.org/en/Release_Notes_GeoGebra_5.0\#New_Command_Line_Arguments
} 


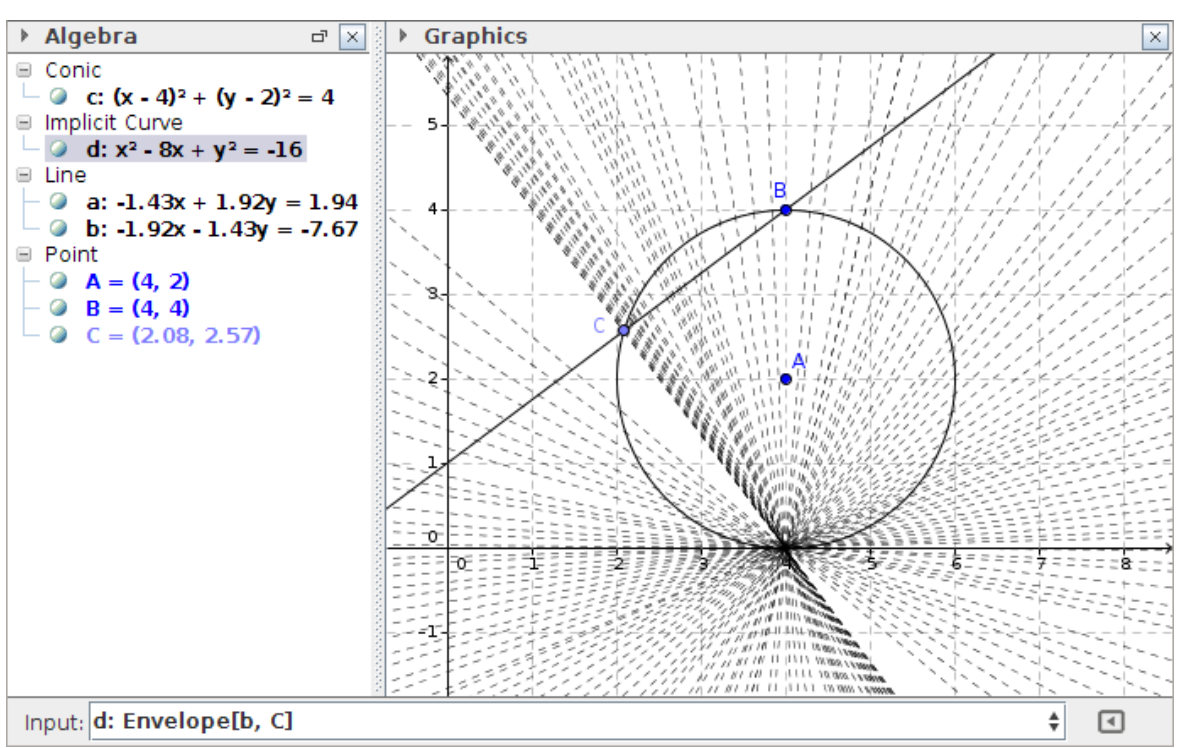

Fig. 3 The envelope of a simple family of lines.

the line $x=4$ is returned, that is a false result since this line comes from a degeneration when $C=B$ (see Section 3.2). The true envelope consists of the point $(4,0)$, as it is computed by the new Envelope command ${ }^{10}$ and shown as an implicit curve.

Online Resource 3 is a video of an envelope computation where three SingularWS instances are used: the first one calls a locally installed instance through VirtualBox while the others use the canonical address and a remote Raspberry $\mathrm{Pi}$, respectively. The connection to remote servers was done via ADSL, suggesting that no significant timing differences with the local instance exist if the canonical SingularWS is used. The construction (see Figure 4) consists of a circle with center $A(5,2)$ and passing through $B(5,4)$, a point $C$ on it, and the family of lines $C F$, where $F$ is another point on the circle at a fixed distance from $C$ (this distance is controlled with points $D$ and $E$ by means of the Compass tool). The Envelope command, when applied to a line $a=C F$ and the point $C$, returns the implicit curve $e$. Note that replacing line $C F$ by $C G$ identical result will be obtained, since $F$ and $G$ are algebraically indistinguishable points. Furthermore, if the distance from $C$ is set to 4 (that is, both circles in the figure are tangent), the points $F$ and $G$ coincide, and the envelope is exactly the point $A$, as GeoGebra indicates through the implicit curve $x^{2}-10 x+y^{2}-4 y+29=(x-5)^{2}+(y-2)^{2}=0$. Currently, GeoGebra allows users to select an undefined object as the line $a$ in this case for computing the envelope. Finally, if the distance is greater than 4, the envelope remains defined, while not having real points. Future GeoGebra versions will

10 http://wiki.geogebra.org/en/Envelope_Command 


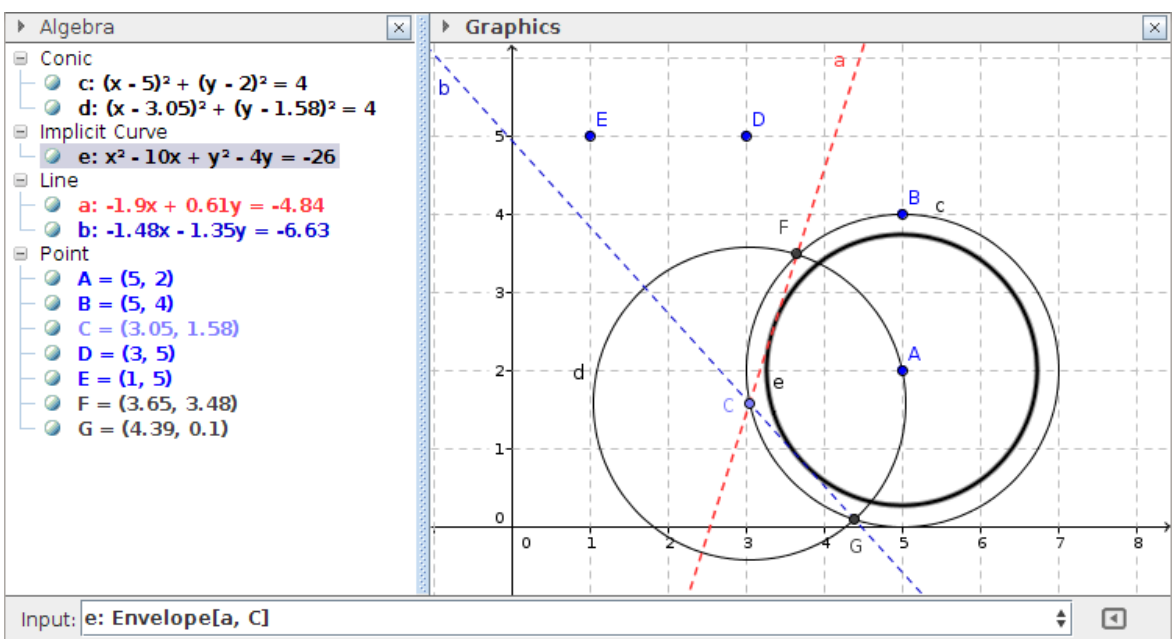

Fig. 4 The envelope of lines $C F$ when $C$ moves along the circle $c$.

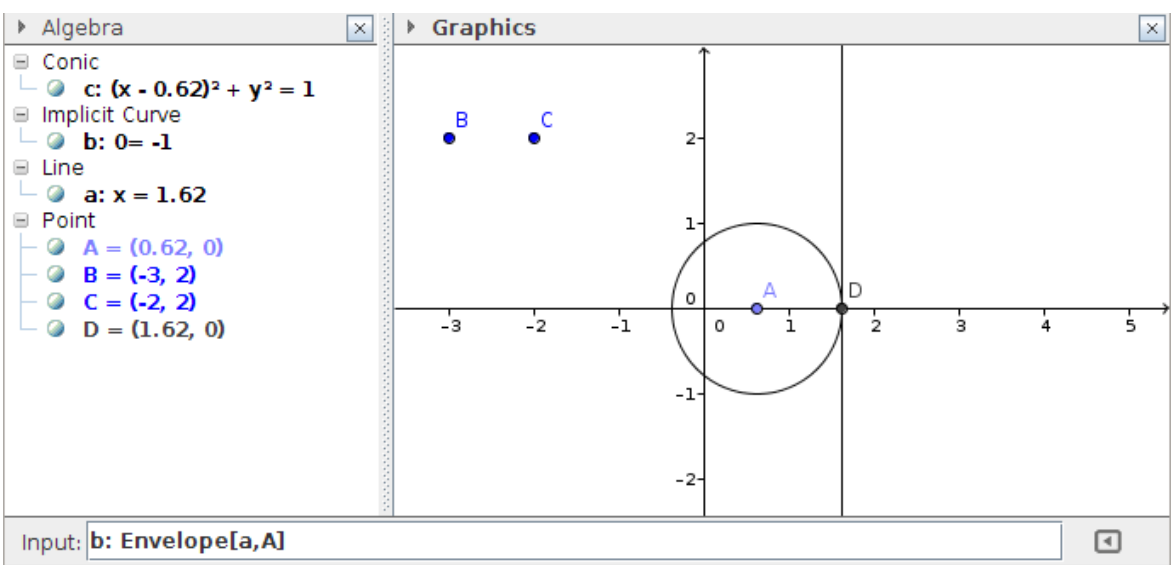

Fig. 5 The envelope of the vertical lines through the moving point $D$ does not exist.

ban the use of undefined objects, and checks for testing imaginary objects will be implemented.

Both envelopes above described are included as GeoGebra constructions in Online Resource 4, together with other illustrative examples.

There are families of lines which do not have envelope. Consider a vertical line at fixed distance of a moving point in the horizontal axis. Let $A\left(x_{1}, x_{2}\right)$ be the point on the axis $\left(x_{2}=0\right)$ and set the distance to 1 (line $x=x_{1}-1$ ). The parametric system $\left\{x-x_{1}+1, x_{2},-1\right\}$ has no solution, and therefore the envelope does not exist. In such cases, GeoGebra returns the implicit curve $0=-1$ (see Figure 5 and Online Resource 4 for construction download).

Finally, since GeoGebra can compute envelopes, it also can deal with offsets, caustics,... Figure 6 shows the 1 -offset of the ellipse $x^{2}+5 y^{2}=5$. Note 


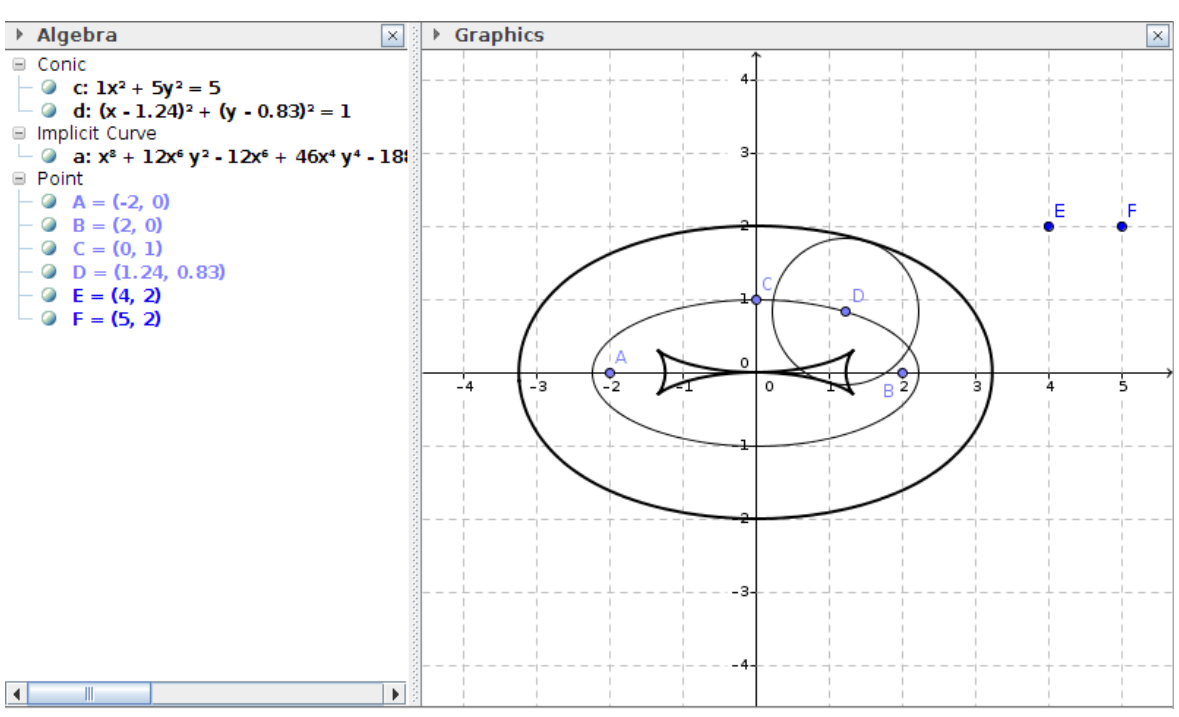

Fig. 6 The 1-offset of an ellipse.

that currently the conic must be defined through the Ellipse command. If a user defines the ellipse as an implicit curve through the Input window and asks for the envelope, an internal exception is declared and the new Envelope object is labeled as undefined. The reason of this behavior is that the current data structure of GeoGebra does not yet include implicit curves as paths of points parametrizing families of curves. Note also that any user trying to compute caustics should not use GeoGebra commands related to reflection, since they do not yet give adequate answers for SingularWS.

\subsection{Future extensions}

An obvious work will consist of extending the described GeoGebra abilities to $3 \mathrm{D}$ loci and envelopes. Preliminary work on such computations in spatial DG has been discussed in [20], and the ongoing development of GeoGebra3D should allow such extension.

A common task for both 2 and 3D environments in GeoGebra will be extending the current data structure to efficiently manage an algebraic description of constructions. Besides completing the definition of GeoGebra commands to use with SingularWS (recall the above limitations of reflection and caustics), plotting procedures need to accommodate to objects that are not anymore described by polynomials, but by constructible sets. And, since the procedures here described work in the complex field, decisions on unveiling non real results should be implemented. These rules will come on a twofold basis: theoretical results from the mathematical side and pedagogical experiences with DG users. 


\section{Conclusions}

We described the design and implementation of SingularWS, a web service allowing access to Singular, a computer algebra system for polynomial computations. By using a Singular algorithm for parametric polynomial systems solving, new GeoGebra commands dealing with geometric loci and envelopes are illustrated. These commands extend current dynamic geometry abilities, returning new knowledge about the structure of loci and envelopes. Although the service has been primarily designed for GeoGebra use, its free open source character allows an easy connection with any other environment. As a consequence, it is argued that this new knowledge demands a new data structure for representing objects in dynamic geometry development.

Acknowledgements The authors want to thank Antonio Montes for his personal comments on the GC algorithm and Miguel Abánades for his constructions involving envelopes.

\section{References}

1. Sutherland, I.E., Sketchpad: A man-machine graphical communication system, Tech. Rep. 574, Computer Laboratory, University of Cambridge (2003)

2. Gao, X.S, Lin, Q.: MMP/Geometer - A software package for automated geometric reasoning, Lect. Notes Artif. Int. 3763, 44-66 (2006)

3. Gao, X.S., Zhang, J.Z., Chou, S.C.: Geometry Expert. Nine Chapters, Taiwan (1998)

4. Recio, T., Vélez, M.P.: Automatic discovery of theorems in elementary geometry, J. Autom. Reasoning 23, 63-82 (1999)

5. Botana, F., Valcarce, J.L.: A dynamic-symbolic interface for geometric theorem discovery, Comput. Educ. 38, 21-35 (2002)

6. Janičić, P.: Geometry constructions language, J. Autom. Reasoning 44, 3-24 (2010)

7. Decker, W., Greuel, G.M., Pfister, G., Schönemann, H.: Singular - A computer algebra system for polynomial computations, http://www.singular.uni-kl.de (2013). Accessed 27 December 2013

8. Montes, A., Wibmer, M.: Groebner bases for polynomial systems with parameters, J. Symb. Comput. 45, 1391-1425 (2010)

9. Oracle Corporation: Java Native Interface (2013)

10. Ancsin, G., Hohenwarter, M., Kovács, Z.: GeoGebra Goes Web, The Electronic J. of Math. \& Technology 7 (6) (2013)

11. Botana, F., Kovács Z., Weitzhofer, S.: Implementing theorem proving in GeoGebra by using a Singular webservice. In Sendra, J.R., Villarino, C. (eds.) Proceedings EACA 2012, pp. 67-70. Universidad de Alcalá, Alcalá de Henares (2012)

12. Kovács, Z., Parisse, B.: Giac and GeoGebra — improved Groebner basis computations, Special Semester on Applications of Algebra and Number Theory, Workshop 3 on Computer Algebra and Polynomials https://www.ricam.oeaw.ac.at/specsem/specsem2013/workshop3/slides/parisse-kovacs.pdf (2013). Accessed 27 December 2013

13. Kovács, Z., Singular WebService, VirtualBox ISO Image, http://ggb1.idm.jku.at/ kovzol/VMs/SingularWS-20140104.zip (2014). Accessed 7 January 2014

14. Kovács, Z.: Singular WebService in GeoGebra, http://dev.geogebra.org/trac/wiki/SingularWS (2012). Accessed 27 December 2013

15. Kovács, Z.: Singular WebService documentation and source code, http://code.google.com/p/singularws/source/browse/doc/README (2012). Accessed 27 December 2013 
16. Chen, X., Wang, D.: Management of geometric knowledge in textbooks, Data Knowl. Eng. 73, 43-57 (2012)

17. Botana, F., Abánades, M.A.: Automatic deduction in (dynamic) geometry: Loci computation, Comp. Geom-Theor. Appl. 47, 75-89 (2014)

18. Botana, F.: Interactive versus symbolic approaches to plane loci generation in dynamic geometry environments, Lect. Notes Comput. Sc 2330, 211-218 (2002)

19. Bruce, J.W., Giblin, P.J.: Curves and Singularities. Cambridge University Press, Cambridge (1984)

20. Botana, F.: A parametric approach to 3D dynamic geometry, Math. Comput. Simulat. (2013) doi:10.1016/j.matcom.2012.12.004 